\title{
Mouse Models of Colorectal Cancer and Liver Metastases
}

\author{
M.W. Heijstek O. Kranenburg I.H.M. Borel Rinkes \\ Department of Surgery, Medical Centre Utrecht, Utrecht, The Netherlands
}

\section{Key Words}

Colorectal cancer · Liver metastases - Mouse model •

Tumor model · Optical imaging $\cdot$ Small animal imaging

\begin{abstract}
Colorectal cancer (CRC) is one of the most common malignancies in the western world. Its high mortality rates are particularly related to the occurrence of liver metastases. Many mouse models have been developed to evaluate the various features of CRC in human. Since none of the existing mouse models mimics all the characteristics of human CRC, it is of crucial importance that the optimal model is chosen for each experiment to resolve a specific experimental question. Currently used mouse models for CRC include chemically induced CRC models, genetically engineered mouse models and models in which colon tumors are implanted in recipient mice. Recently, conditional mouse models have been created in which a gene of interest can be (in)activated in a time- and tissue-specific manner. All models have their advantages and limitations. This review highlights the most commonly used mouse models for CRC and its liver metastases, their usefulness and shortcomings, as well as recent improvements, particularly regarding intravital (tumor) imaging.
\end{abstract}

Copyright (C) 2005 S. Karger AG, Basel

\section{Introduction}

With 940,000 recorded cases worldwide per year, colorectal cancer (CRC) is the third most common malignancy in the world. Despite efforts to improve prevention and therapy, nearly 500,000 patients die from CRC each year [1]. It has become evident that the main problem in the treatment of CRC is not so much eradication of the primary tumor, but rather the formation of incurable metastases. Mortality is particularly associated with the occurrence of metastases in the liver.

Animal models for human CRC can provide insight into the mechanisms that underlie the development and pathogenesis of CRC. The ideal animal model should therefore faithfully replicate all aspects of tumor development in man. These include the (sequential) acquirement of genetic alterations with consequent changes in cell behavior and tumor biology. In addition, the metastatic potential and the characteristic sensitivity to therapeutics should ideally be conserved [2]. Furthermore, the tumor model should be practical: tumor take should be predictable and consistent, with a high incidence of affected animals in a narrow time frame [3]. Unfortunately, although a number of models approximate some of the characteristics of human CRC, none meet all these criteria. Therefore, each specific experimental issue should be studied by choosing the model best suited to resolve that particular issue. This review highlights the mouse models cur-

I.H.M. Borel Rinkes, MD

Department of Surgery (G04-228), UMC-Utrecht

PO Box 85500

NL-3508 GA Utrecht (The Netherlands)

Tel. +31 30250 8074, Fax +31 30254 1944, E-Mail I.H.M.BorelRinkes@chir.azu.nl 
rently used for CRC and its liver metastases. The advantages and limitations of these models, recent improvements and remaining challenges are addressed.

\section{Models of Spontaneous and Chemically Induced Colorectal Cancer}

Mouse models of spontaneous and carcinogen-induced CRC are the earliest models described. Spontaneous formation of colorectal cancer in mice occurs with an incidence of $<1 \%[4,5]$ making it impossible to perform experimental studies on CRC development. Although inbred CRC-prone mice develop the disease at a young age, the predictability and reproducibility of tumor take are insufficient for routine experimental use. In addition, the formation of metastases occurs only sporadically in these mice [6].

In order to increase the incidence of CRC, new models were developed in which mice are exposed to carcinogens. Most carcinogens cause malignancies in multiple organs, but some predominantly induce CRC. Frequently used carcinogens are dimethylhydrazine (DMH) and its metabolite azoxymethane (AOM), N-methyl-N-nitro$\mathrm{N}$-nitrosoguanidine (MNNG) and N-methyl-N-nitrosourea (MNU) [6]. The incidence of CRC development depends on the carcinogen used, the dosage, the duration and frequency of administration, as well as on the routing and timing of administration. DMH and AOM can be administered via four routes: oral, subcutaneous, intrarectal and intramuscular. The effectiveness of various carcinogens in inducing colorectal cancer has been compared [6]. Oral dosing of DMH in rats results in a low tumor incidence (14 to 30\%). Subcutaneous administration of $\mathrm{DMH}$ or $\mathrm{AOM}$ results in a colon tumor incidence varying from 0 to $100 \%$. Intrarectal administration of $\mathrm{DMH}$ induces mild hyperplasia and preneoplastic lesions in the colon after 34 weeks. Repeated intramuscular injections of AOM resulted in $80 \%$ incidence of CRC after 12 weeks. Oral dosing of direct-acting MNU induces tumors along the entire gastrointestinal tract, with a decreasing incidence from stomach to rectum. Intrarectal administration of MNU resulted in a colon tumor incidence of $100 \%$. Moreover, metastases developed in 23 to $31 \%$ of mice [7]. In addition, the sex, age and genetic background of the mice affect the incidence of CRC development. Moreover, the intestinal flora, the diet and the immunological status of the mice can interfere with the metabolism of carcinogenic compounds and thereby influence their effective local concentration.
Ideally, all of these variables should be standardized in order to obtain a protocol with which CRC development can be reproducibly induced. A major disadvantage of carcinogen-induced CRC development is the low incidence of tumor take, making it necessary to use large numbers of animals. Moreover, these models are not suitable for studying metastasis formation as this occurs very slowly and infrequently. However, carcinogen-induced CRC development in mice is ideal for studying the influence of diet on tumor development.

\section{Molecular Genesis of Colorectal Cancer}

Multistage carcinogenesis from local hyperplasia to adenoma to invasive carcinoma and metastatic disease requires the sequential mutation of various genes. Four classes of genes are of importance in carcinogenesis: the growth-promoting proto-oncogenes (dominant), the growth-inhibiting cancer-suppressor genes (recessive), genes that regulate apoptosis (dominant or recessive) and genes that regulate repair of damaged DNA. The DNA repair genes affect cell survival or proliferation indirectly by influencing the ability to repair nonlethal damage in other genes, including the proto-oncogenes and the tumor-suppressor genes. A defect in the DNA repair genes predisposes to mutations in the genome and hence to neoplastic transformation.

'Gatekeeper' genes directly influence the growth of cells. Mutations in these genes can initiate the entry into the multistep carcinogenesis pathway. In contrast, 'caretaker' genes affect genomic stability. The mismatch repair genes belong to the latter category.

Although most colorectal cancers in human are regarded as sporadic, a small percentage is due to an autosomaldominant inherited syndrome [1]. These patients carry a germ-line mutation of either a gatekeeper or a caretaker gene; therefore, they have an increased risk of developing cancer. Hereditary nonpolyposis colon cancer (HNPCC) is the most common form of hereditary colon cancer, accounting for $5-8 \%$ of all colon cancers [8]. Colon cancer develops as a result of mutations in a caretaker gene. Familial adenomatous polyposis (FAP) accounts for less than $1 \%$ of all colon cancers [9]. As a result of a mutation in a gatekeeper gene multiple adenomas develop in the intestinal tract, which eventually progress to malignant colon tumors. 


\section{Mouse Models for HNPCC}

Hereditary non-polyposis coli (HNPCC) is a syndrome caused by the inactivation of DNA mismatch repair genes (e.g. MLH-1, MSH-2 and -6, PMS-1 and -2). The tumors are characterized by instability at short tandem repeat sequences, also called microsatellites. Models based on the use of genetically modified mice have contributed greatly to our understanding of the complicated process of tumour development. Mice homozygously deleted for Mlh-1 or MSH-2 develop lymphomas but are also prone to intestinal neoplasia and therefore represent a good model for studying HNPCC development. Mlh1 ${ }^{-/-}$mice develop gastrointestinal tumors in 33\%. Moreover, addition of an APC gene mutation into the $\mathrm{Mlh}^{-/}$mice resulted in a 40-fold increase in the number of GI tumors, leading to $100 \%$ GI tumor formation. There were no reports of metastases in these mice [10]. All homozygous Msh2-deficient mice succumbed to disease within the first year of observation, with lymphomas observed in at least $80 \%$ of the cases. The majority (70\%) of animals 6 months or older developed intestinal neoplasms associated with APC inactivation [11-13]. These models have given insight into the mechanisms underlying the development of CRC. Mice homozygous for mismatch repair (MMR) genes and heterozygous for a defect in the gatekeeper gene APC have shown that the MMR gene enhances APC-mediated intestinal carcinogenesis. Exposure of MMR-deficient cells to endogenous or exogenous mutagens may potentiate tumorigenesis and may be critical in the organ selection in HNPCC $[10,11]$. These models also revealed that MMR-deficient cells fail to induce apoptosis in response to alkylating agents. In contrast, the alkylated base damage remains in the DNA thereby potentiating carcinogenesis. This may have direct implications for chemotherapy of HNPCC patients [14].

\section{Mouse Models for FAP}

As mentioned before, a sequence of specific genetic changes underlies the development of intestinal carcinogenesis in the mouse. These include inactivation of the tumor suppressor genes APC, p53 and Smad3 and activation of the proto-oncogene K-Ras. Mice carrying specific deletions of the $A P C$ gene develop multiple adenomas throughout the gastrointestinal tract, especially in the small intestine [15], but there is no tumor development in the colon. In other models, for example the Multiple intestinal neoplasia (Min) mouse models, additional so- matic changes need to be induced by applying carcinogens for high incidence intestinal tumor development. Mice heterozygous for the APC gene have been used to study the general principles of carcinogenesis, to test the response to suppressive agents such as aspirin $[16,17]$ and to test the carcinogenicity of various compounds [18].

Inactivation of the p53 gene mainly leads to lymphomagenesis, although colon tumors can develop after administration of colon-specific carcinogens $[6,19]$. Whereas the $A P C$ mice can be used for studying the early stages of intestinal polyp formation, the p53 knockout mouse is particularly suited for studying the influence of diet, carcinogens and chemotherapeutics on tumor progression. Studies in p53-deficient mice have revealed that absence of p53 interferes with the physiology of apoptotic cell death, i.e. cell cycle arrest after DNA damage is failing. It has been shown that several chemotherapeutics, such as ectopiside, adriamycin and 5-fluorouracil, can induce p53-mediated apoptosis. Tumors that retained wild-type p53 were responsive to these therapies, in contrast to tumors derived from $\mathrm{p} 53$ null cells. These findings indicate that human cancer patients with p53 mutations in a tumor might be resistant to these therapies [20].

TGF $\beta$-related growth factors have also been implicated to play a role in tumor formation. TGF $\beta$ transduces its signal into the cell via the second messengers Smad2, Smad 3 and Smad4. It has recently been shown that Smad3 mutant mice develop metastatic colorectal cancer. All inbred Smad $3^{-/-}$mutants had colorectal adenocarcinomas, some of which were highly aggressive and approximately one-third invaded through all layers of the bowel wall. In several mutant mice lymph nodes were enlarged and showed infiltration by carcinoma [21].

\section{Conditional Gene (In)Activation to Model FAP}

Germline transmission of disrupted tumor suppressor genes or activated oncogenes can create cancer-prone mice. Such mice have proven to be powerful tools for studying gene function in tumor biology. For example, the different $A P C$ mutant mice faithfully model familial adenomatous polyposis coli, but are less suitable for studying sporadic CRC, as the initiating mutation is transmitted through the germline. In addition, tumors may develop too early for experimental therapeutics, causing high mortality rates at a young age. New techniques have made it possible to induce time-controlled and tissue-specific somatic gene activation or inactivation [22-28]. An example of such a model is the Apc ${ }^{\text {loxP }}$ mouse in which exon 14 of the APC gene is flanked by 
loxP sites. The gene can be deleted by local infection of the colorectal region with an adenovirus expressing the Cre recombinase [29]. Likewise, conditional expression of $\beta$-catenin (the transcriptional coactivator that is targeted for degradation by APC) leads to the formation of gastrointestinal polyps [30, 31].

The adenomas that develop in the mouse models described above show proper resemblance to the equivalent lesions in FAP patients. Furthermore, the (mostly intestinal) tumors form spontaneously, predictably and with a high incidence. They are nonimmunogenic and arise in immunocompetent mice (in contrast to the implantation models described later). A disadvantage is that metastases form infrequently (if at all) and unpredictably. However, if they form, they do so spontaneously thereby resembling the process in cancer patients [32].

\section{Advantages, Limitations and Applications of the} Genetically Engineered Mouse Models

The genetically engineered mouse models are used primarily to determine whether a particular gene is involved in the pathogenesis of cancer, or whether a gene product, involved in specific signalling processes, contributes to cancer in combination with other predisposing conditions. Because the development of colorectal cancer in these models is not predictable and metastases seldom develop, they are not often used in drug testing [3]. Despite their shortcomings, such models can be used to study the effects of early therapeutic intervention or preventive measures. Such studies are likely to be predictive of the clinical outcome, because they represent the natural history of tumour development. For example, a comparison was made between the efficacy of agents on the prevention of CRC in mice and polyp recurrence in humans [18]. NSAIDs strongly decrease the tumor yield in the small intestine of Min mice. This is consistent with epidemiological studies suggesting that NSAIDs might decrease the colorectal cancer incidence in humans.

With the development of the conditional gene (in)activation models it has become possible to generate highly specific tumors in a narrow time window with high incidence [26]. These models provide new opportunities to establish the influence of specific (combinations of) genes in the initiation and progression of cancer. Conditional mouse models will be ideally suited to study drugs that interfere with specifically mutated regulatory pathways promoting tumor growth [26]. The potential of this approach can be illustrated by an experiment in which tumor cells with amplified c-myc (an oncogene) expression and wild-type p53 are susceptible to 5-FU, in con- trast to tumor cells with a mutated p53 gene. In accordance with these findings, retrospective analysis of a phase III clinical trial showed that only patients with tumours containing amplified expression of c-myc and p53 responded to therapy with 5-FU [33].

Disadvantages of these models are insufficient expression in the presence of the inducer (poor inducibility) or unwanted expression in the absence of the inducer (leakiness). As a result, expression of the target gene may not be $100 \%$ tissue specific [24].

\section{Implantation Models}

Grafts from either human (xenografts) or murine (allografts) tumors can be implanted into recipient mice. To prevent xenograft rejection, either nude mice or mice with severe combined immunodeficiency (SCID) are used. In nude mice the $N u$ gene is knocked out, resulting in hairless thymus-less mice which cannot generate $\mathrm{T}$ lymphocytes. SCID mice have a mutation in the cytokine (IL-2) receptor resulting in disrupted lymphocyte maturation and a deficit in circulating, mature, functional $\mathrm{T}$ and $B$ cells [34]. In contrast to their lack of adaptive immunity, SCID mice possess a completely intact innate immune system, with normal numbers of macrophages, natural killer cells and granulocytes. Activation of the innate immune system in SCID mice is responsible for a complex immune response against human xenografts. The host-versus-graft response can vary considerably between mice and, by inference, varies between samples from different tumors. By selective elimination of tumor samples or by blocking the host-versus-graft reaction the variability may be reduced.

Numerous murine tumor cell lines have been established from either spontaneously occurring tumors or from carcinogen-induced tumors. These cell lines may be propagated in vitro or as subcutaneous tumors in mice. Tumor tissue or a tumor cell suspension can be implanted or injected into syngeneic mice, either at ectopic sites (mostly subcutaneously) or at orthotopic sites (e.g. CRC cells in the colon).

\section{Intact Colon Cancer Tissue}

Human colorectal tumor biopsies can be transplanted directly into recipient nude mice and serve as models for human metastatic colon cancer [35-38]. The grafted tumor tissue will thus grow out in a microenvironment that best mimics the original microenvironment. A major disadvantage of using intact tumor tissue is the intrinsic het- 
erogeneous nature of the material, both within a tumor, as well as between different tumors. This makes standardization and comparison between various experiments difficult. Furthermore, human tumor tissue often contains large necrotic areas that are not suitable for experimentation [37].

\section{Ectopic and Orthotopic Tumor Models}

There are several methods for implanting human tumor cells or tumor tissue (table 1). By far the easiest and most frequently used model is subcutaneous injection/ implantation. The accessibility of subcutaneous tumors is a tremendous advantage for monitoring tumor progression and for assessing the effects of therapeutic intervention. However, a major disadvantage is that the subcutaneous (ectopic) microenvironment greatly differs from that of the colon or the liver. Interactions between the host environment and the tumor graft determine tumor cell expression profiles, the levels of growth factors and nutrients, as well as tumour angiogenesis and metastatic behavior. For instance, $\mathrm{CRC}$ growth in the colon will give rise to metastases, whereas subcutaneous growth does not [35, 37, 39-41].

Alternatively, tumor growth at orthotopic sites (i.e. the colon, the cecum or the rectal wall) can be achieved by subserosal injection of tumor cells, or by surgical orthotopic implantation (SOI) of tumor fragments. These fragments (typically $1 \mathrm{~mm}^{3}$ ) can be obtained either from biopsies or from previously grown tumors and are sutured onto the cecum, the colon or the rectal wall. For successful attachment of the implant, it is recommended to damage the serosal lining of the intestinal wall using a surgical blade prior to implantation. All of these procedures are carried out with the aid of a microscope.

Orthotopic and ectopic organ environments differentially influence the sensitivity of tumor cells to chemotherapeutics [37]. For example, colon cancers grown subcutaneously were more susceptible to doxorubicin than tumors growing in the cecum of mice [42].

\section{Models for Liver Metastasis Formation}

The preferred site of metastasis formation in CRC patients is the liver. Because any therapy of cancer is invariably the therapy of metastatic disease, it is essential that models in which CRC actually forms, (liver) metastases develop as well. Surgical orthotopic implantation of highly metastatic tumor cells in the colon of nude mice has been shown to result in the rapid and efficient formation of metastases in liver (100\%), lymph nodes and spleen $[35,36,43-45]$. This model is ideal for studying many
Table 1. Implantation models of colorectal cancer

$$
\text { Implanted specimen Implantation method }
$$

Cell lines or cell suspension
Ectopic injection (subcutaneous)

Orthotopic injection in the colon, cecum or rectal wall

Intraperitoneal injection

Injection into specific blood vessels, usually the portal vein

Solid tumor grafts
Intrasplenic injection
Ectopic injection (subcutaneous)

Surgical orthotopic implantation (SOI) in the colon, cecum or rectal wall aspects of metastasis formation as well as for analyzing the efficacy of novel therapeutic agents. Liver metastases in the mouse can also be induced by intrasplenic or direct intraportal injection of colon cancer cells. As the tumor cells reach the hepatic microcirculation, they become arrested either by size restriction or by specific retention. Arrested cells may grow out while still within the vasculature or they may extravasate and colonize the liver parenchyma. Although a small percentage of injected cells eventually manage to grow out and form local tumors, the overall efficiency of (liver) metastasis formation is low.

The metastatic potential of a given tumor graft or a cell line can be augmented by so-called 'passage cycles'. During this procedure the liver metastases are isolated, expanded in vitro and are subsequently re-injected into the spleen or the vena porta. The resulting liver metastases can then be re-isolated. With each selection cycle the metastatic potential of the cells increases [46]. This also means that the cell populations selected by this procedure have become genetically distinct from the original cell line and should be treated as independent subclones.

The above techniques result in the colonization of the entire liver with single tumor cells. However, for some types of experiments, it may be required to grow tumors in a single liver lobe or even as a solitary tumor. To obtain tumor growth in an isolated liver lobe we usually perform subcapsular injection of tumor cell suspensions. A single liver tumor may be obtained by implantation of tumor fragments from pre-established (subcutaneous) tumors. During this procedure, a small incision in the liver parenchyma is made, followed by rapid implantation of the fragment. This usually stops most of the bleeding, but further hemostasis may be achieved by applying gentle pressure at the wound surface using cotton. For example, 
this intrahepatic model has been used to study the efficacy of 5-fluorocytosine/cytosine deaminase enzyme prodrug gene therapy. Cells expressing cytosine deaminase activate the prodrug 5-FC into the cytotoxic agent 5-FU. This approach has shown to inhibit intrahepatic CRC tumor progression [47].

A major disadvantage of the experimental metastasis models is that only late stages of metastasis formation can be studied. The early stages, including local invasion at the site of the primary tumor and gaining access to lymphatics or blood vessels, are by-passed by direct injection of tumor cells into the portal vein or into the spleen. Proper modeling of the early phases of spontaneous metastasis formation requires growing a metastatic tumor orthotopically (i.e. in the colon). A disadvantage of this method is the poor efficiency and predictability of spontaneous metastasis formation from an orthotopic primary tumor. Nonetheless, models involving spontaneous metastasis formation have been used to test the efficacy of experimental therapeutics in preventing metastasis. Hepatotropic liposomal adriamycin (hLip-ADM) has been shown to effectively inhibit liver metastasis $(0$ vs. $83 \%$ in control animals) from orthotopically implanted colon cancer [48]. As an alternative method to study early metastasis formation, noninvasive imaging techniques may be used to follow intraportally injected cells through the early phases of cell arrest, extravasation, colonization and growth (see below).

\section{Advantages, Limitations and Applications of the Implantation Models}

The advantages of the implantation models are the high degree of predictability and rapidity of tumor formation. The major advantage of studying s.c. growing tumors is the possibility to screen large numbers of drugs. The models require minimal labor, are relatively inexpensive, rapid and reproducible. Limitations are the inefficient formation of metastases and the danger of scoring false-positive results when testing anticancer drugs in experimental therapies. Tumors growing subcutaneously may show unnatural reactions to experimental drugs [37, 42], possibly because the expression profile of multidrug resistance $(m d r)$ genes is altered $[49,50]$. Thus, therapeutic results obtained in a subcutaneous tumor model should always be followed by studies in an orthotopic implantation model, preferably one in which metastases develop [51].

Drugs that modulate the tumor microenvironment cannot be evaluated by ectopic models and must be studied in orthotopic models. Compounds that activate the immune system should be tested in immunocompetent mice. Furthermore, it is necessary to standardize protocols with respect to the route, the method and the dosing of tumor cell inoculation, as well as that of the therapeutic agents.

The applicability of the various mouse models described is summarized in table 2 .

\section{Imaging and Monitoring Tumor Growth and Metastasis Formation}

\section{Histological and Molecular Profiling}

To what extent do CRC tumors in mouse models resemble the tumors in CRC patients? Several modern molecular biological techniques may be applied to answer this question. It is expected that the analyses of gene expression and protein abundance profiles will become extremely important in tumor typing for diagnostic purposes. These techniques are also best suited for comparing human tumor behavior in patients and in mice. Techniques that have been used to date include comparisons of histological and chromosomal parameters [52].

Histological analyses can also be applied for quantifying tumor growth postmortem. The outgrowth of CRC liver metastases is routinely determined by measuring the area of liver tissue that has been replaced by tumor tissue. Histological analysis of tumor growth has two major disadvantages. First, analyses can only be performed once, postmortem. Second, it is time-consuming and laborious. These two disadvantages can be overcome by using modern noninvasive imaging techniques.

\section{Noninvasive Imaging Techniques}

Noninvasive imaging techniques allow monitoring of tumor initiation, progression and response to therapy in a single animal by sequential imaging analysis. Conventional imaging methods that are used in the clinic have been adjusted to visualize murine tumors $[53,54]$ including magnetic resonance imaging (MRI), computed tomography (CT), positron emission tomography (PET) and single photon emission tomography (SPECT). Furthermore, fluorescence and bioluminescence imaging techniques allow for monitoring the growth of tumors expressing either Aequorea victoria green fluorescent protein (GFP) or firefly luciferase.

\section{EGFP and Fluorescence Imaging}

Ultrasensitive charge-coupled device (CCD) cameras can detect tumor cells that continuously express EGFP at 
Table 2. Applications of the various mouse models of colorectal cancer

\begin{tabular}{|c|c|}
\hline Mouse model & Experimental applications \\
\hline Chemically induced mouse model & Dietary influences on carcinogenesis \\
\hline $\begin{array}{l}\text { Conventional transgenic } \\
\text { mouse model }\end{array}$ & $\begin{array}{l}\text { Function of gene products and the consequences of } \\
\text { their expression on normal processes } \\
\text { Involvement of particular genes in carcinogenesis } \\
\text { (in combination with other predisposing } \\
\text { conditions) } \\
\text { Influence of carcinogens and chemopreventive } \\
\text { agents on colorectal carcinogenesis } \\
\text { Influence of early intervention therapies on } \\
\text { (colorectal) cancer }\end{array}$ \\
\hline $\begin{array}{l}\text { Conditional transgenic } \\
\text { mouse models }\end{array}$ & $\begin{array}{l}\text { Analyzing drugs that interfere with specifically } \\
\text { mutated regulatory pathways driving tumour } \\
\text { growth } \\
\text { Establishing the influence of specific genes and } \\
\text { combinations of genes in the initiation and } \\
\text { progression of cancer }\end{array}$ \\
\hline \multicolumn{2}{|l|}{ Implantation models } \\
\hline $\begin{array}{l}\text { Ectopic } \\
\text { Orthotopic }\end{array}$ & $\begin{array}{l}\text { Screening large amounts of cytotoxic agents } \\
\text { Verifying the effects of experimental therapies } \\
\text { found in ectopic mouse models } \\
\text { Analyzing the pathogenesis of metastases } \\
\text { Assessing the effect of therapies on metastasis } \\
\text { Evaluating drugs that adjust the tumour-host } \\
\text { interaction }\end{array}$ \\
\hline Syngenic mice & Evaluating immune therapy \\
\hline
\end{tabular}

high concentrations. The development of CRC liver metastases has been imaged noninvasively by this method [55-57]. One of the drawbacks of this technique is that the high level E-GFP expression that is required for noninvasive imaging may interfere with the growth and survival of a number of CRC tumor cell lines. Another limitation of using fluorescence imaging based on E-GFP expression remains the difficulty of detecting and quantifying the fluorescence emitted from GFP-expressing tumors through the abdominal wall, due to scattering and light absorption. The latter problem can largely be prevented by creating a flap of skin that may be opened whilst imaging and closed afterwards for multiple measurements of tumor growth in a single mouse. Alternatively, the tumor-bearing tissue can be exposed surgically and in this way it is possible to visualize local tumor-tissue interaction, tumor progression, angiogenesis and metastasis formation at the single cell level. Although technically possible, ethical considerations make it undesirable to perform multiple invasive imaging analyses on a single mouse. Despite the disadvantages outlined above it is expected that with modern laser scanning technologies and improved detection limits and signal-to-noise ratios, it will be possible to follow the growth of (auto)fluorescent tumors noninvasively on a routine basis.

\section{Luciferase and Bioluminescence Imaging}

An alternative to following tumor growth by fluorescence is to make use of bioluminescence. To this end, tumor cells must be engineered to express luciferase, a photoprotein that generates light following oxidative conversion of its substrate luciferin. A disadvantage of this system is that prior to imaging, the mice must receive an intraperitoneal injection of luciferin. Low-light photoncounting cameras can subsequently detect the light emitted from the tumor cells (fig. 1). This technique is ideal for visualizing tumor growth in a noninvasive and sequential manner and it has successfully been used to study the growth of CRC cells in the mouse liver (fig. 2). Moreover, chemotherapeutic effects on intrahepatic CRC were easily assessed by this method [47].

In conclusion, optical imaging (bioluminescence and fluorescence) allows monitoring of tumor growth, progression and metastasis formation as well as the response 


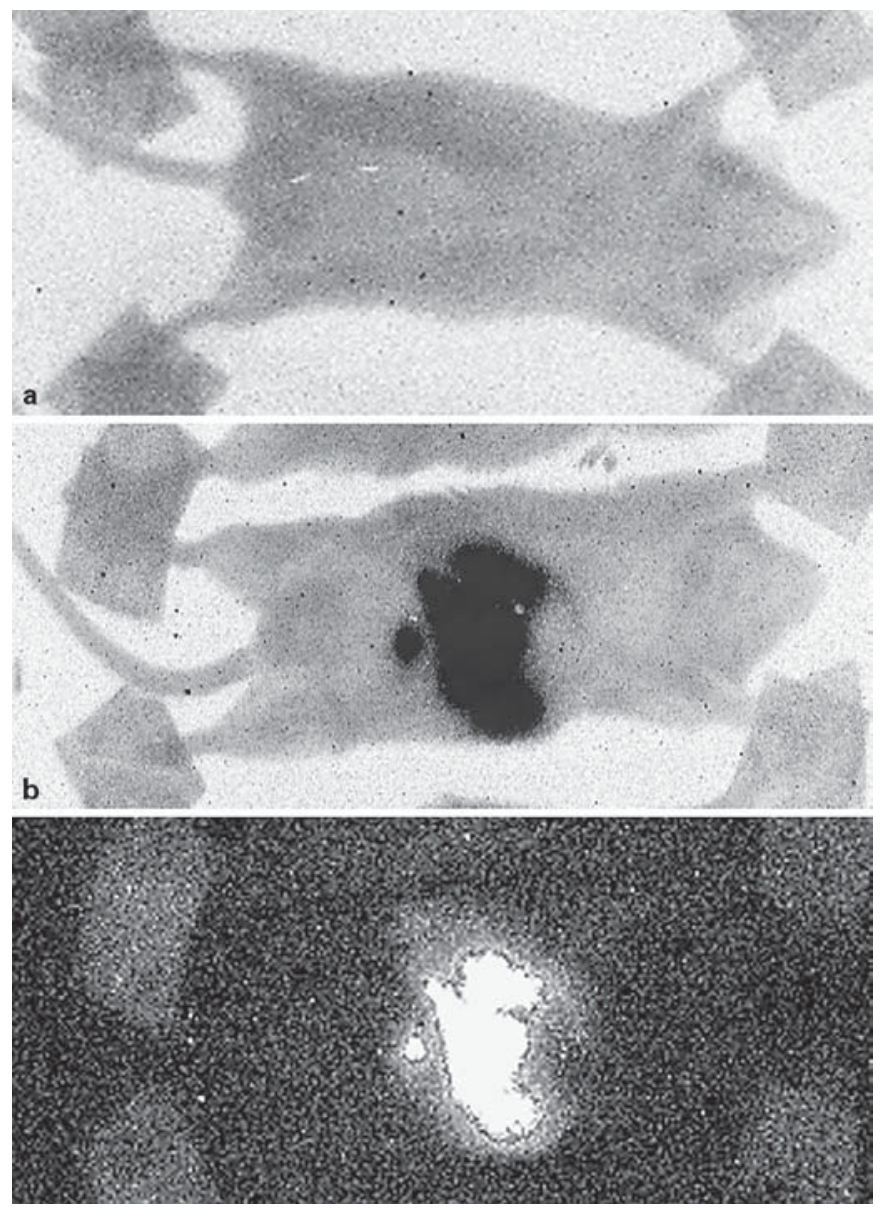

Fig. 1. Detection of liver metastases by luciferase imaging. Liver metastases were induced in a single liver lobe by intrahepatic injection of $5 \cdot 10^{5}$ luciferase expressing colorectal cancer cells. Ten days after injection, tumour growth was analysed by luciferase imaging. Before (a) and 5 min after (b) luciferin injection. of the tumor to therapy. When compared to classical (twodimensional) histological analyses, the imaging techniques show improved (three-dimensional) accuracy and reproducibility as well as the enormous advantage of serial measurements. Fewer animals are therefore required to collect statistically reliable results [26].

\section{Additional Considerations in Modeling Cancer in Mice}

There are few notorious differences between mice and human and confounding variables that influence carcinogenesis. These differences make accurate modeling of cancer difficult and careful interpretation of the mouse models is therefore essential.

An important issue is telomeres, structures at the chromosome ends that protect chromosomes against damage. Telomere exhaustion results in apoptosis [58]. Telomerase activity prevents telomere shortening, thereby allowing immortal growth of cells. Humans do not have functional telomerase in their cells, in contrast to mice. Through knockout of the gene encoding the telomerase enzyme ( $\mathrm{Terc}^{-/}$mice), mouse models with more humansized telomeres can be created [59].

Another point meriting attention is age-dependent carcinogenesis. Cancer is predominantly a disease of the elderly. In contrast, cells divide less frequently in older mice, which probably makes the mice less susceptible to tumor initiation and progression. A study in a Min/+ mouse model revealed that younger mice were more sus-
Light image

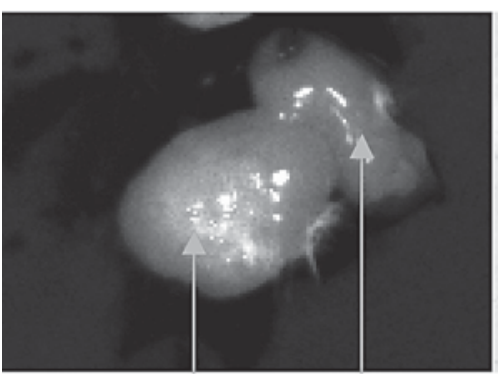

Hepatic tumour growth confined to 1 liver lobe

Normal parenchyma
Luciferase overlay image

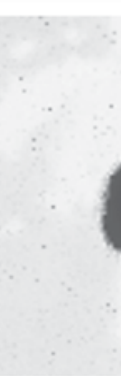
residual liver

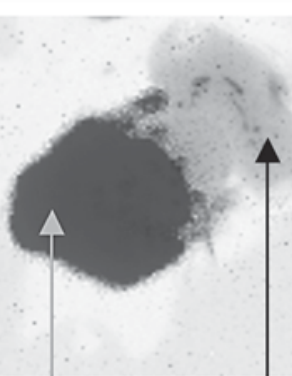

Luciferase expressing tumour

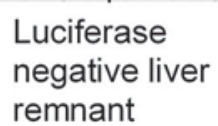

\section{Luciferase image}

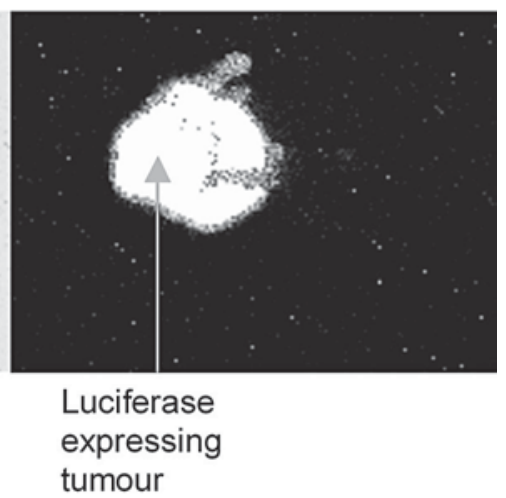

Fig. 2. Luciferase imaging of exteriorized liver. Fourteen days after intrahepatic injection of $5 \cdot 10^{5}$ luciferase expressing colorectal cancer cells, the mice were injected with luciferin and killed after $5 \mathrm{~min}$. The liver was immediately isolated and analyzed by luciferase imaging. 
ceptible to AOM-induced colorectal carcinogenesis than older mice [60].

Dietary factors and physical activity may also influence carcinogenesis. Dietary components may be carcinogenic [61], or may improve cellular defense mechanisms [62]. Dietary restriction may have an anticarcinogenic effect [63], and limited physical activity might increase the incidence of colorectal malignancies [64].

For adequate interpretation and comparability it is crucial to standardize these factors. By doing so, existing differences between the many different laboratories may be diminished.

\section{Conclusions}

The ideal animal model does not yet exist, although a mouse model can imitate parts of the human carcinogenesis. Mouse models can only be used as predictors of outcome. Therefore, it is crucial to use the optimal model to resolve a specific experimental question (table 2).
Chemical-induced models can be used to study dietary influences on carcinogenesis. The genetically engineered mouse models can be applied to study the influence of genetic changes on carcinogenesis and the interaction of these mutations with environmental factors. The ectopic implantation models can be used to screen a large number of cytotoxic agents. To verify the effects of experimental therapies found in ectopic mouse models, to evaluate drugs adjusting the tumor-host interaction, to study therapy for metastasis and to analyze the pathogenesis of metastases, the orthotopic implantation models are necessary. Syngeneic implantation models are suitable for immune therapy experiments.

Standardization of protocols used and adequate description of techniques used is equally important, in order to make comparison between various experiments and laboratories possible. Novel imaging and molecular tools will further improve efficacy, accuracy and reproducibility of mouse models. By combining the (latest) mouse models with sophisticated imaging technologies it might be possible to accelerate the development of colorectal cancer therapies.

\section{References}

1 Parkin DM, Bray F, Ferlay J, Pisani P: Estimating the world cancer burden: Globocan 2000. Int J Cancer 2001;94:153-156.

$\checkmark 2$ Hann B, Balmain A: Building 'validated' mouse models of human cancer. Curr Opin Cell Biol 2001;13:778-784.

$\checkmark 3$ Rosenberg MP, Bortner D: Why transgenic and knockout animal models should be used (for drug efficacy studies in cancer). Cancer Metast Rev 1998;17:295-299.

4 Anisimov VN, Zabezhinski MA, Rossolini G, Zaia A, Piantanelli A, Basso A, Piantanelli L: Long-live euthymic BALB/c-nu mice. II. Spontaneous tumors and other pathologies. Mech Age Dev 2001;122:477-489.

$\checkmark 5$ Chandra M, Frith CH: Spontaneous neoplasms in aged CD-1 mice. Toxicol Lett 1992;61:6774.

6 Kobaek-Larsen M, Thorup I, Diederichsen A, Fenger C, Hoitinga MR: Review of colorectal cancer and its metastases in rodent models: Comparative aspects with those in humans. Comp Med 2000;50:16-26.

7 Yang J, Shikata N, Mizuoka H, Tsubura A: Colon carcinogenesis in shrews by intrarectal infusion of N-methyl-N-nitrosourea. Cancer Lett 1996;110:105-112.

$>8$ Peltomaki P: Deficient DNA mismatch repair: A common etiologic factor for colon cancer. Hum Mol Genet 2001;10:735-740.
Bulow S, Faurschou NT, Bulow C, Bisgaard ML, Karlsen L, Moesgaard F: The incidence rate of familial adenomatous polyposis. Results from the Danish Polyposis Register. Int $\mathrm{J}$ Colorectal Dis 1996;11:88-91.

$>10$ Edelmann W, Yang K, Kuraguchi M, Heyer J, Lia M, Kneitz B, Fan K, Brown AM, Lipkin M, Kucherlapati R: Tumorigenesis in Mlh1 and Mlh1/Apc1638N mutant mice. Cancer Res 1999;59:1301-1307.

11 de Wind N, Dekker M, van Rossum A, van der Valk M, te Riele H: Mouse models for hereditary nonpolyposis colorectal cancer. Cancer Res 1998;58:248-255.

12 Prolla TA, Baker SM, Harris AC, Tsao JL, Yao $\mathrm{X}$, Bronner CE, Zheng B, Gordon M, Reneker J, Arnheim N, Shibata D, Bradley A, Liskay RM: Tumour susceptibility and spontaneous mutation in mice deficient in Mlh1, Pms 1 and Pms2 DNA mismatch repair. Nat Genet 1998; 18:276-279

13 Reitmair AH, Redston M, Cai JC, Chuang TC, Bjerknes M, Cheng H, Hay K, Gallinger S, Bapat B, Mak TW: Spontaneous intestinal carcinomas and skin neoplasms in Msh2-deficient mice. Cancer Res 1996;56:3842-3849.

14 de Wind N, Dekker M, Berns A, Radman M, te Riele $\mathrm{H}$ : Inactivation of the mouse Msh2 gene results in mismatch repair deficiency, methylation tolerance, hyperrecombination, and predisposition to cancer. Cell 1995;82: $321-330$.
15 Fodde R, Edelmann W, Yang K, van Leeuwen C, Carlson C, Renault B, Breukel C, Alt E, Lipkin M, Khan PM: A targeted chain-termination mutation in the mouse Apc gene results in multiple intestinal tumors. Proc Natl Acad Sci USA 1994;91:8969-8973.

16 Williamson SL, Kartheuser A, Coaker J, Kooshkghazi MD, Fodde R, Burn J, Mathers $\mathrm{JC}$ : Intestinal tumorigenesis in the Apc1638N mouse treated with aspirin and resistant starch for up to 5 months. Carcinogenesis 1999;20: 805-810.

17 Jacoby RF, Seibert K, Cole CE, Kelloff G, Lubet RA: The cyclooxygenase- 2 inhibitor celecoxib is a potent preventive and therapeutic agent in the min mouse model of adenomatous polyposis. Cancer Res 2000;60:5040-5044.

18 Corpet DE, Pierre F: Point: From animal models to prevention of colon cancer. Systematic review of chemoprevention in min mice and choice of the model system. Cancer Epidemiol Biomarkers Prev 2003;12:391-400.

19 Donehower LA: The p53-deficient mouse: A model for basic and applied cancer studies. Semin Cancer Biol 1996; 7:269-278.

20 Macleod KF, Jacks T: Insights into cancer from transgenic mouse models. J Pathol 1999; 187:43-60.

21 Zhu Y, Richardson JA, Parada LF, Graff JM: Smad3 mutant mice develop metastatic colorectal cancer. Cell 1998;94:703-714. 
22 Clarke AR: Manipulating the germline: Its impact on the study of carcinogenesis. Carcinogenesis 2000;21:435-441.

23 Jackson-Grusby L: Modeling cancer in mice. Oncogene 2002;21:5504-5514.

-24 Jonkers J, Berns A: Conditional mouse models of sporadic cancer. Nat Rev Cancer 2002;2: 251-265.

25 Lewandoski M: Conditional control of gene expression in the mouse. Nat Rev Genet 2001;2 743-755.

26 Meuwissen R, Jonkers J, Berns A: Mouse models for sporadic cancer. Exp Cell Res 2001;264: 100-110.

-27 Tuveson DA, Jacks T: Technologically advanced cancer modeling in mice. Curr Opin Genet Dev 2002;12:105-110.

28 Van Dyke T, Jacks T: Cancer modeling in the modern era: Progress and challenges. Cell 2002;108:135-144.

-29 Shibata H, Toyama K, Shioya H, Ito M, Hirota M, Hasegawa S, Matsumoto H, Takano H, Akiyama T, Toyoshima K, Kanamaru R, Kanegae Y, Saito I, Nakamura Y, Shiba K, Noda T: Rapid colorectal adenoma formation initiated by conditional targeting of the Apc gene. Science 1997;278:120-123.

- 30 Harada N, Tamai Y, Ishikawa T, Sauer B, Takaku K, Oshima M, Taketo MM: Intestinal polyposis in mice with a dominant stable mutation of the beta-catenin gene. EMBO J 1999; 18:5931-5942.

- 31 Harada N, Miyoshi H, Murai N, Oshima H, Tamai Y, Oshima M, Taketo MM: Lack of tumorigenesis in the mouse liver after adenovirus-mediated expression of a dominant stable mutant of beta-catenin. Cancer Res 2002;62: 1971-1977.

-32 Kerbel RS: What is the optimal rodent model for anti-tumor drug testing? Cancer Metast Rev 1998;17:301-304.

- 33 Arango D, Corner GA, Wadler S, Catalano PJ, Augenlicht LH: c-myc/p53 interaction determines sensitivity of human colon carcinoma cells to 5 -fluorouracil in vitro and in vivo. Cancer Res 2001:61:4910-4915.

34 Bankert RB, Egilmez NK, Hess SD: HumanSCID mouse chimeric models for the evaluation of anti-cancer therapies. Trends Immunol 2001:22:386-393.

- $35 \mathrm{Fu}$ X, Herrera H, Kubota T, Hoffman RM: Extensive liver metastasis from human colon cancer in nude and scid mice after orthotopic onplantation of histologically-intact human colon carcinoma tissue. Anticancer Res 1992; 12:1395-1397.

- $36 \mathrm{Fu}$ XY, Besterman JM, Monosov A, Hoffman RM: Models of human metastatic colon cancer in nude mice orthotopically constructed by using histologically intact patient specimens. Proc Natl Acad Sci USA 1991;88:9345-9349.

- 37 Pocard M, Tsukui H, Salmon RJ, Dutrillaux B, Poupon MF: Efficiency of orthotopic xenograft models for human colon cancers. In Vivo 1996; 10:463-469.
38 Kuo TH, Kubota T, Watanabe M, Furukawa T, Teramoto T, Ishibiki K, Kitajima M, Moossa AR, Penman S, Hoffman RM: Liver colonization competence governs colon cancer metastasis. Proc Natl Acad Sci USA 1995;92: 12085-12089.

39 Bresalier RS, Raper SE, Hujanen ES, Kim YS: A new animal model for human colon cancer metastasis. Int J Cancer 1987;39:625-630.

40 Giavazzi R, Campbell DE, Jessup JM, Cleary K, Fidler IJ: Metastatic behavior of tumor cells isolated from primary and metastatic human colorectal carcinomas implanted into different sites in nude mice. Cancer Res 1986;46:19281933.

41 Kubota T: Metastatic models of human cancer xenografted in the nude mouse: The importance of orthotopic transplantation. J Cell Biochem 1994;56:4-8.

-42 Wilmanns C, Fan D, O’Brian CA, Bucana CD, Fidler IJ: Orthotopic and ectopic organ environments differentially influence the sensitivity of murine colon carcinoma cells to doxorubicin and 5-fluorouracil. Int J Cancer 1992;52: 98-104.

43 Furukawa T, Kubota T, Watanabe M, Kuo TH, Nishibori H, Kase S, Saikawa Y, Tanino $\mathrm{H}$, Teramoto T, Ishibiki K: A metastatic model of human colon cancer constructed using cecal implantation of cancer tissue in nude mice. Surg Today 1993;23:420-423.

-44 Rashidi B, Sun FX, Jiang P, An Z, Gamagami R, Moossa AR, Hoffman RM: A nude mouse model of massive liver and lymph node metastasis of human colon cancer. Anticancer Res 2000;20:715-722.

45 Sun FX, Sasson AR, Jiang P, An Z, Gamagami R, Li L, Moossa AR, Hoffman RM: An ultrametastatic model of human colon cancer in nude mice. Clin Exp Metastasis 1999;17:4148.

46 Morikawa K, Walker SM, Jessup JM, Fidler IJ: In vivo selection of highly metastatic cells from surgical specimens of different primary human colon carcinomas implanted into nude mice. Cancer Res 1988;48:1943-1948.

47 Nyati MK, Symon Z, Kievit E, Dornfeld KJ, Rynkiewicz SD, Ross BD, Rehemtulla A, Lawrence TS: The potential of 5-fluorocytosine/cytosine deaminase enzyme prodrug gene therapy in an intrahepatic colon cancer model. Gene Ther 2002;9:844-849.

48 Matsuda I, Konno H, Tanaka T, Nakamura S: Antimetastatic effect of hepatotropic liposomal adriamycin on human metastatic liver tumors. Surg Today 2001;31:414-420.

49 Dong Z, Radinsky R, Fan D, Tsan R, Bucana $\mathrm{CD}$, Wilmanns C, Fidler IJ: Organ-specific modulation of steady-state mdr gene expression and drug resistance in murine colon cancer cells. J Natl Cancer Inst 1994;86:913920 .
-50 Fidler IJ, Wilmanns C, Staroselsky A, Radinsky R, Dong Z, Fan D: Modulation of tumor cell response to chemotherapy by the organ environment. Cancer Metast Rev 1994;13:209222.

-51 Killion JJ, Radinsky R, Fidler IJ: Orthotopic models are necessary to predict therapy of transplantable tumors in mice. Cancer Metast Rev 1998;17:279-284.

52 Resor L, Bowen TJ, Wynshaw-Boris A: Unraveling human cancer in the mouse: Recent refinements to modeling and analysis. Hum Mol Genet 2001;10:669-675.

-53 Lewis JS, Achilefu S, Garbow JR, Laforest R, Welch MJ: Small animal imaging. Current technology and perspectives for oncological imaging. Eur J Cancer 2002;38:2173-2188.

-54 Weissleder R: Scaling down imaging: Molecular mapping of cancer in mice. Nat Rev Cancer 2002;2:11-18.

55 Chishima T, Miyagi Y, Wang X, Yamaoka H, Shimada H, Moossa AR, Hoffman RM: Cancer invasion and micrometastasis visualized in live tissue by green fluorescent protein expression. Cancer Res 1997;57:2042-2047.

56 Hoffman RM: Orthotopic transplant mouse models with green fluorescent protein-expressing cancer cells to visualize metastasis and angiogenesis. Cancer Metast Rev 1998;17:271277.

57 Yang M, Baranov E, Jiang P, Sun FX, Li XM, Li L, Hasegawa S, Bouvet M, Al Tuwaijri M, Chishima T, Shimada H, Moossa AR, Penman S, Hoffman RM: Whole-body optical imaging of green fluorescent protein-expressing tumors and metastases. Proc Natl Acad Sci USA 2000; 97:1206-1211.

58 Herzig M, Christofori G: Recent advances in cancer research: Mouse models of tumorigenesis. Biochim Biophys Acta 2002;1602:97113.

59 Blasco MA: Telomeres in cancer and aging: Lessons from the mouse. Cancer Lett 2003; 194:183-188.

60 Paulsen JE, Steffensen IL, Namork E, Eide TJ, Alexander J: Age-dependent susceptibility to azoxymethane-induced and spontaneous tumorigenesis in the Min/+ mouse. Anticancer Res 2003;23:259-265.

61 Wasan HS, Novelli M, Bee J, Bodmer WF: Dietary fat influences on polyp phenotype in multiple intestinal neoplasia mice. Proc Natl Acad Sci USA 1997;94:3308-3313.

62 Minamoto T, Mai M, Ronai Z: Environmental factors as regulators and effectors of multistep carcinogenesis. Carcinogenesis 1999;20:519527.

63 Hursting SD, Perkins SN, Phang JM, Barrett JC: Diet and cancer prevention studies in $\mathrm{p} 53-$ deficient mice. J Nutr 2001;131(suppl):3092S3094S.

-64 Dreosti IE: Nutrition, cancer, and aging. Ann NY Acad Sci 1998;854:371-377. 